\title{
EXPERIMENT ON THE EFFECT OF SUPERFICIAL GAS-LIQUID VELOCITIES ON SLUG FLOW-INDUCED VIBRATION IN AN INCLINED SAGGED RISER
}

\author{
Bowen Maa ${ }^{a}$ Narakorn Srinila ${ }^{*}$, Hongjun Zhu ${ }^{b}$, Yue Gao ${ }^{b}$ \\ aMarine, Offshore \& Subsea Technology Group, School of Engineering, Newcastle University, \\ Newcastle Upon Tyne, United Kingdom \\ bState Key Laboratory of Oil \& Gas Reservoir Geology \& Exploitation, Southwest Petroleum University, \\ Chengdu, China
}

\begin{abstract}
Subsea liquid-gas flows conveyed through a flexible riser or pipeline may develop into various flow patterns including slug flow. In this study, the slug flow-induced vibration (SIV) of an inclined sagged riser conveying upward air-water flows is experimentally investigated. A small-scale experiment is carried out in an air-water test loop with a section of a freehanging catenary tube made of silica gel. Attention is placed on the effect of superficial gas and liquid velocities on SIV responses. Both pipe motions and flow patterns are recorded using non-intrusive high-speed cameras. Pressure variations are also measured at the pipe inlet and outlet by two pressure transducers. The SIV system is tested by employing different ratios of the superficial gas-liquid velocities. Occurrence of unstable slug flows is captured at the relatively high gas-toliquid velocity ratios, leading to a large-amplitude SIV. Experimental results of the space-time varying riser responses and oscillation frequencies are reported together with the associated slug flow features. Depending on the gas-liquid superficial velocities, slug flow characteristics are observed to vary significantly. These entail an intermittent SIV with modulated amplitudes and frequencies along riser span, signalling a potential dynamic stress and fatigue-related concern. In all experimental cases, the riser responses are found to be multi-modal and dominated by the fundamental planar mode whereas an out-of-plane vibration is negligible. Experimental observations suggest the key interrelationships of the two-fluid flow conditions, the slug characteristics and the pipe dynamics. This finding is meaningful for a practical design of riser transporting internal multiphase flows.

Keywords: slug flow-induced vibration, catenary riser, fluid-structure interaction, multiphase flow, experiment.
\end{abstract}

\footnotetext{
*Corresponding author: narakorn.srinil@newcastle.ac.uk
}

\section{INTRODUCTION}

Subsea risers are widely used in the offshore oil and gas industry for deep-water productions where hydrocarbon flows transported from the subsea fields are typically multi-phased. Liquid-gas flows may appear in several flow patterns during the operation. Characterized by the alternating liquid- and gasdominant regions, slug flows are practically problematic due to a fluctuation in the flow inertia, momenta and pressure potentially causing slug flow-induced vibration (SIV). When the slug flow is transported through the pipe bends, elbows or highly curved risers, the space-time varying loads may be further enhanced due to the change in the flow direction. In subsea oil \& gas applications, cyclic and excessive stresses due to SIV may affect the integrity/reliability of subsea systems. In [1], the sagged risers are recognized to be more vulnerable to SIV owing to the associated time-space varying slug flow weight, centrifugal and Coriolis forces, especially when the slug flow frequency matches with the structural frequency giving rise to a resonance and amplified response. Recently, numerical predictions of SIV features have been carried out for different pipe geometries [2-4] and various external excitations [5-7]. Nevertheless, experimental investigation into slug flowinduced vibrations is still lacking in the literature.

Gas-liquid superficial velocities are recognized as the key parameters governing the formation of slug flows and the consequent structural dynamics. By considering the horizontal pipe transporting air-water flows under various phase velocity conditions, SIV has been experimentally investigated by some researchers [8]. The correlation between slug flow velocities and SIV of a horizontal pipe was highlighted in [9]. This study suggested that the increasing slug unit velocity and slug liquid length are responsible for amplified responses. For a horizontal pipe with multiple supports, Liu and Wang [10] reported the time-varying natural frequencies of the flexible pipe owing to the unsteady slug flows. For a given superficial liquid velocity, 
they found a critical gas superficial velocity depending on the pipe length and flexural rigidity. Recently, the role of slug frequency in determining dynamic stresses was studied by Mohmmed et al. [11] who pointed out the relationship between slug frequencies and superficial phase velocities.

Recently, SIV of curved pipes has been experimentally studied by Zhu et al. [12] who observed slug flow-induced planar vibrations and a resonant frequency between the pipe and the internal pressure fluctuation. They remarked the strong dependence of SIV on the ratios of gas-liquid flow rates and the associated liquid slug lengths. For a highly curved pipe in a lazy-wave shape and subject to slug flows, Vieiro et al. [13] experimentally observed pipe responses due to a severe slugging flow. By varying the gas flow rate for a fixed liquid flow rate, the internal pressure, riser top tension and SIV were measured. It was found that the severe slugging cases lead to more pronounced SIV than the stable slug flow.

In this study, experiments of an inclined sagged, catenary riser transporting upward air-water flows are carried out under various liquid-gas flow conditions, primarily aiming to explore the effects of superficial gas-liquid velocities on SIV responses and associated slug flow characteristics.

\section{EXPERIMENTAL SETUP}

The experiment is carried out through an air-water test loop of the State Key Laboratory of Oil and Gas Reservoir Geology and Exploitation at Southwest Petroleum University, China. The experiment facility is schematically shown in Fig. 1. The test loop starts with the devices supplying air and water into separated flowlines. An air compressor with a maximum flow rate up to 450 litre/minute is employed to supply the dry air (at $1 \mathrm{~atm}$ and $20^{\circ} \mathrm{C}$ ), followed by a float flowmeter allowing the maximum measurement of 3 litre/minute with an accuracy of $1 \%$. The water flowline is supplied through a centrifugal pump with a maximum flow rate of 1.5 litre/minute The water is dyed into the black colour distinguishing itself from the air for the sake of slug flow characterization. The water flow rate is measured through an electromagnetic flowmeter up to 1.3 litre/minute with $0.5 \%$ accuracy.

As shown in Fig. 2a, individual air-water flowlines meet at a mixing point via a pneumatic tee adapter. The two-phase flow is then transported through a downward pipe (Fig. 1). This arrangement is imposed to induce a stratified flow, which is a dominant flow pattern in downward two-phase flow [14], initiating the slug flow development $[13,15]$. The air-water flow is then conveyed through a long horizontal part $(2 \mathrm{~m})$ enabling the fully developed slug flow traveling through the inclined sagged riser test section with fixed ends. The loop ends with the separation of air-water flows by the gravity, discharging the air into the atmosphere and recycling the water into the storage tank. The flexible catenary pipe is made of silica gel, with 4, 6 and $128 \mathrm{~mm}$ being its internal diameter $(d)$, outer diameter $(D)$ and length $(L)$, respectively. Pipe properties are summarized in Table 1, where the fundamental in-plane natural frequency $\left(f_{n}\right)$ and damping ratio are obtained from free decay tests under air-filled and water-filled pipe conditions.

\section{DATA ACQUISITION PROCESS}

High-speed cameras (Baumer HXG20) are used as in Fig. $2 \mathrm{c}$ as a non-intrusive measurement. To capture the planar SIV and flow regime, one camera is placed in front of the riser test segment, facing the in-plane view whereas another is placed beneath the horizontal part to monitor the flow pattern. Two pressure transducers are installed at the pipe inlet and outlet ends, capturing the pressure variation based on the calibrated gauge pressure of zero $\mathrm{Pa}$.

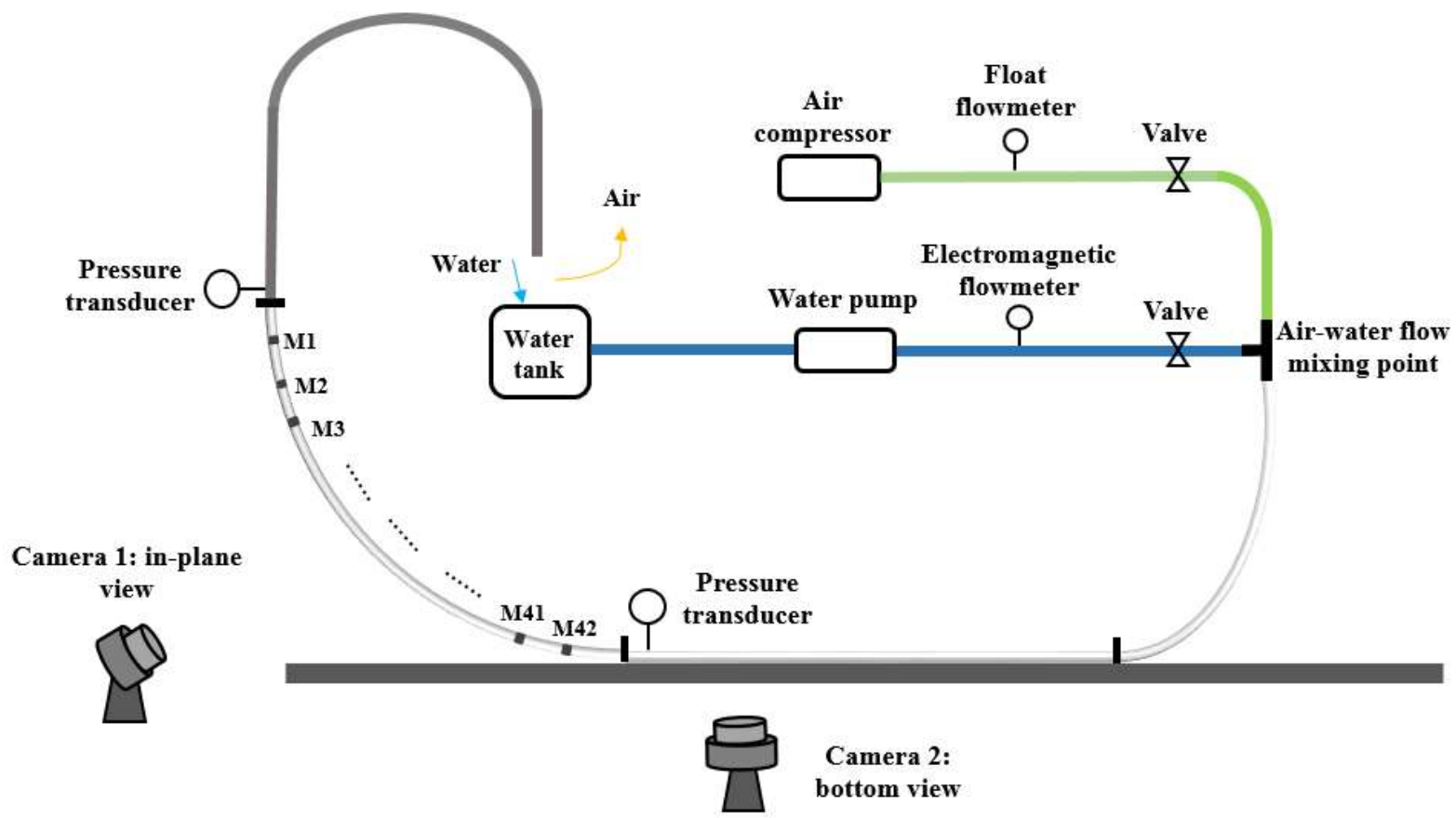

Figure 1. Schematic layout of an air-water test loop for experimental studies on SIV of catenary riser. 
(a)

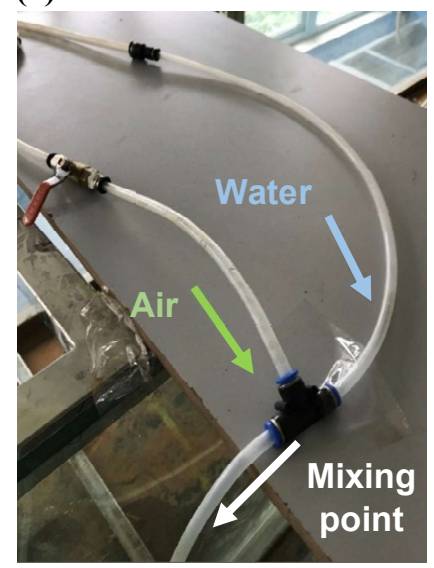

(c)

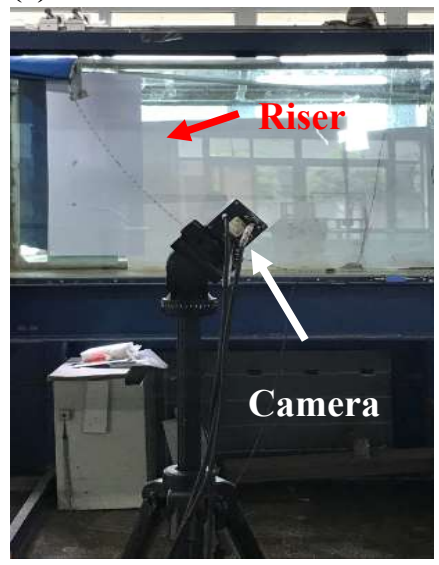

(b)

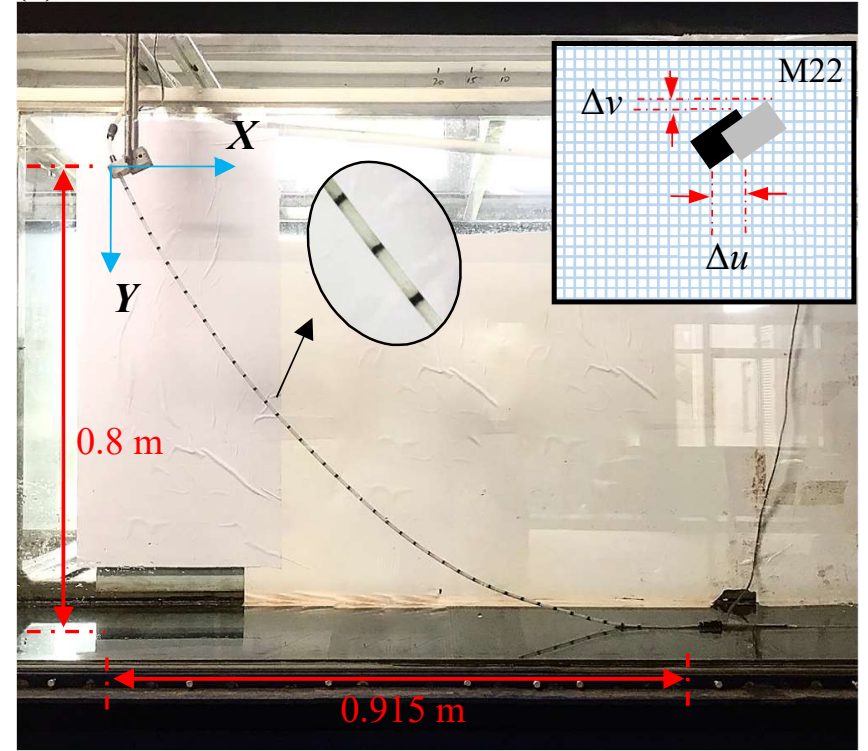

Figure 2. Photographs of (a) air-water flowlines, (b) riser with markers and (c) camera view.

Table 1. Riser pipe properties.

\begin{tabular}{|c|c|}
\hline Length & $1.28 \mathrm{~m}$ \\
\hline Internal diameter & $0.004 \mathrm{~m}$ \\
\hline Outer diameter & $0.006 \mathrm{~m}$ \\
\hline Elastic modulus & $7.15 \mathrm{MPa}$ \\
\hline Density of the riser & $1041.8 \mathrm{~kg} / \mathrm{m}^{3}$ \\
\hline Horizontal span & $0.915 \mathrm{~m}$ \\
\hline Vertical height & $0.8 \mathrm{~m}$ \\
\hline Natural frequency (air-filled) & $2.72 \mathrm{~Hz}$ \\
\hline Natural frequency (water-filled) & $2.52 \mathrm{~Hz}$ \\
\hline Damping ratio (air-filled) & $0.98 \%$ \\
\hline Damping ratio (water-filled) & $0.86 \%$ \\
\hline
\end{tabular}

High-quality videos at a sampling frequency of $100 \mathrm{~Hz}$ (100 images per second) are recorded in which each image has a resolution of $2048 \times 1088$ pixels. To extract the riser motion from the images, 42 weightless black markers (M1 to M42) of $4 \mathrm{~mm}$ in length are located and equally spaced by a $30 \mathrm{~mm}$ centre-to-centre interval along the riser span (Fig. 2b). The first and last makers are $25 \mathrm{~mm}$ away from the riser ends. Such markers establish the referenced spatial arclength coordinate $(s)$ enabling us to capture overall riser SIV and characterize the slug flow features in terms of length, velocity and frequency.

Prior to the tests, cameras are calibrated for which a static riser layout is taken as a frame of reference for the dynamic response. The system coordinate is set by aligning $Y$ with the gravity and $X$ to the right as illustrated in Fig. 2b. To ensure a fully developed slug flow, the data acquisition is started after a test run of 10 minutes in each case. Riser SIV responses are recorded as consecutive frames over the acquisition time ( $T=$ $80 \mathrm{~s}$ ). By scaling the pixel changes between the two adjacent frames in $X$ and $Y$ directions to the actual distances as $\Delta u$ and $\Delta v$ (Fig. 2), the respective vibration responses ( $u$ and $v$ ) along time $(t)$ at all markers can be achieved and presented.

\section{EXPERIMENTAL RESULTS AND DISCUSSION}

SIV tests are carried out under various flow conditions as listed in Table 2. Five cases are based on different superficial gas velocities $\left(u_{g s}\right)$ for a given liquid velocity $\left(u_{l s}\right)$ (C1-C5), yielding the gas-to-liquid velocity ratio $\left(R_{G L}\right)$ from 1 to 5 . In this section, experimental SIV results and flow regimes are reported and discussed. Cases $\mathrm{C} 3-\mathrm{C} 5$ entail unstable slug flow (US) patterns whereas the relatively uniform air-water flow with a no-slugging (NS) appears in cases $\mathrm{C} 1$ and $\mathrm{C} 2$. The case C5 with the maximum $R_{G L}=5$ is found to produce the most pronounced SIV which is exemplified in the following.

Table 2. Test cases with observed FIV and flow patterns.

\begin{tabular}{|c|c|c|c|c|c|}
\hline Case & $\begin{array}{c}\boldsymbol{u}_{l s} \\
(\mathbf{m} / \mathbf{s})\end{array}$ & $\begin{array}{c}u_{g s} \\
(\mathrm{~m} / \mathrm{s})\end{array}$ & $\boldsymbol{R}_{G L}$ & $\begin{array}{c}\text { Flow } \\
\text { pattern }\end{array}$ & SIV \\
\hline C1 & \multirow{5}{*}{0.774} & 0.774 & 1 & NS & NA \\
\hline $\mathrm{C} 2$ & & 1.548 & 2 & NS & $\mathrm{SA}$ \\
\hline C3 & & 2.322 & 3 & US & SA \\
\hline $\mathrm{C} 4$ & & 3.096 & 4 & US & MA \\
\hline $\mathrm{C} 5$ & & 3.870 & 5 & US & LA \\
\hline
\end{tabular}

Note: NS denotes no-slugging and US denotes unsteady slug flows.

NA, SA, MA LA are negligible-, small-, moderate- and large-amplitude SIV, respectively.

Planar SIV response envelopes in the $\mathrm{C} 5$ case are shown in Fig. 3, exhibiting the fundamental modal oscillation of the catenary riser with double curvatures. The riser SIV is more pronounced in $X$ than $Y$ direction. This may be attributed to the riser geometry relation to the horizontal flow inlet. The $1^{\text {st }}$ mode SIV responses are modulated for which the spatially unsteady responses may be represented by the root-meansquared (RMS) values (red dashed lines). The ratios of the maximum to associated RMS responses are about 1.98 and 1.73 in $X$ and $Y$ direction, respectively. This is relatively far from $(2)^{1 / 2}$ for a simple harmonic motion, implying the occurrence of multi modal riser dynamics. For a given time instant, the instantaneous $u$ and $v$ profiles (yellow dashed lines) are plotted in Fig. 3, showing higher order modal contributions This feature suggests that SIV is a superposition of different vibrational modes, which are directly associated with the slug flow characteristics.

The C5 SIV response is further analysed at some specific pipe/marker locations. Time histories of riser SIV at M14 and M24, which correspond to the maximum and minimum RMS values (Fig. 3), are depicted in Fig. 4. The associated timevarying frequencies are also obtained by conducting a wavelet analysis (Figs. 4b and d). The responses at these two points have a similar pattern despite their considerable amplitude difference. Both signals reveal pronounced oscillations when 
$40<t<50 \mathrm{~s}$, with strongly modulated amplitudes which are expected for the chaotic-like oscillation (Fig. 3). This unsteady SIV can be reflected in the associated oscillation frequency $\left(f_{o}\right)$ in Figs. $4 \mathrm{~b}$ and d. The time-varying $f_{o}$ at both locations account for the observed SIV unsteadiness. For M14, the riser vibrates at a dominating $f_{o} \approx 2.6 \mathrm{~Hz}$, which is close to $f_{n}$. This suggests the resonance occurrence of the fundamental mode, consistent with the oscillation profiles in Fig. 3. It is worth noting that $f_{o}$ falls between $f_{n}$ under air-filled $(2.72 \mathrm{~Hz})$ and $f_{n}$ water-filled
$(2.52 \mathrm{~Hz})$ conditions. This is due to the combined effects of internal fluid, pressure and centrifugal forces on the system mass and stiffness. However, by inspecting Fig. 4b, a deviation of the governing $f_{o}$ from $2.6 \mathrm{~Hz}$ to $2.8 \mathrm{~Hz}$ is observed during $0<t<10 \mathrm{~s}$. The neighbouring frequency contents ranging from $1.5-4 \mathrm{~Hz}$ are found to participate in SIV response, together with the main $f_{o}$. Such frequency distributions and modulations signal an intermittent SIV mechanism.
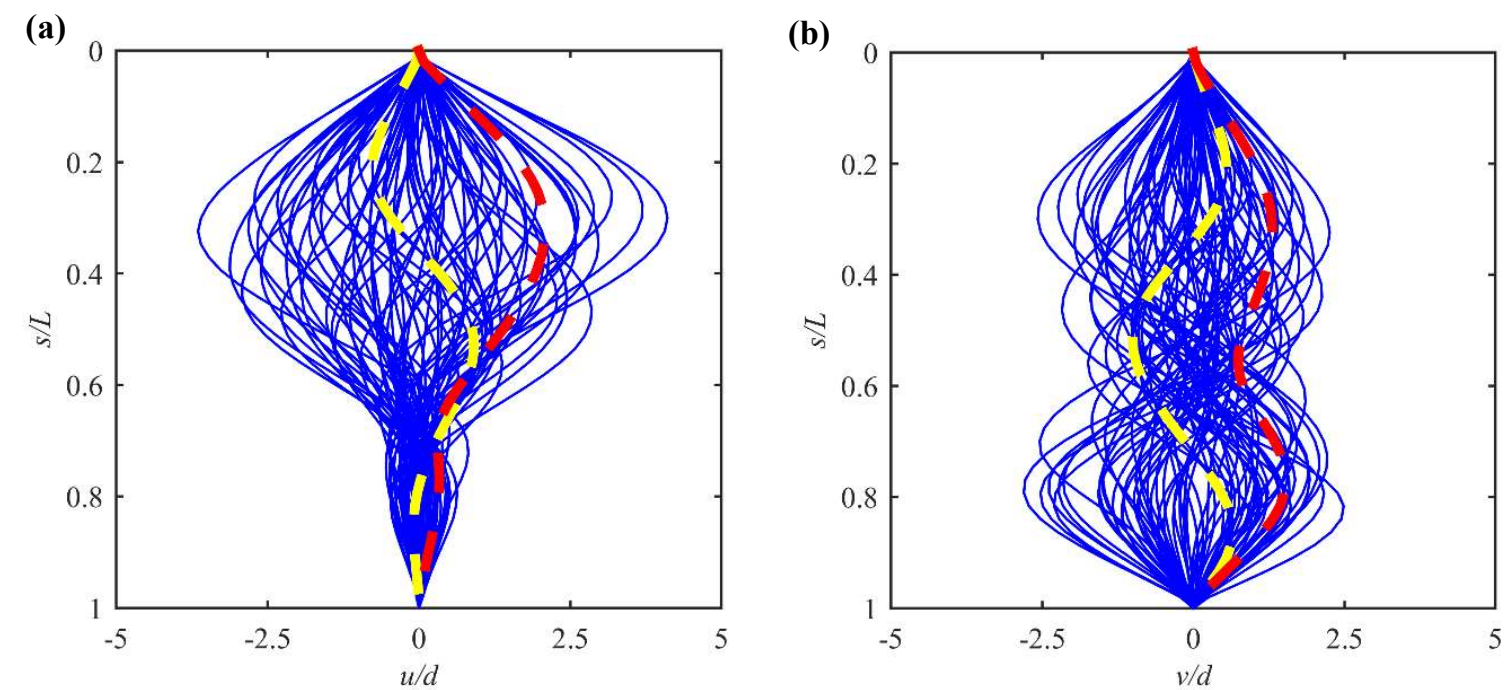

Figure 3. Time-varying spatial profiles (solid blue lines) with associated spatial RMS displacements (dashed red lines) and selected profiles of higher order SIV responses (dashed yellow lines) in (a) $X$ and (b) $Y$ for case C5.

(a)
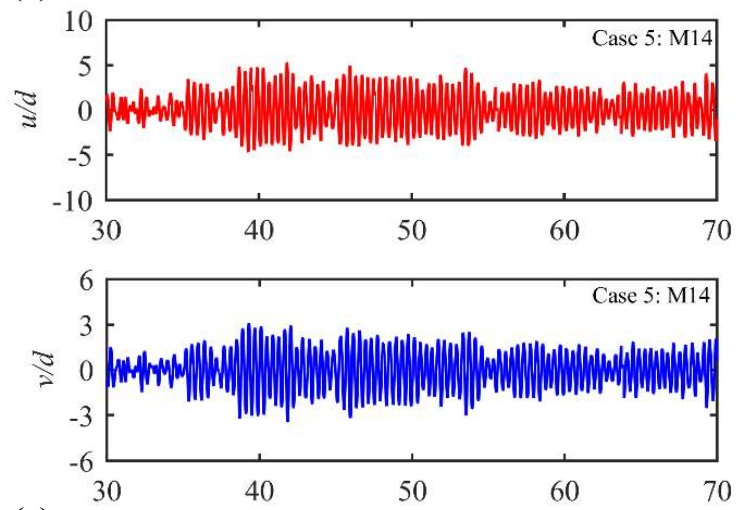

(c)
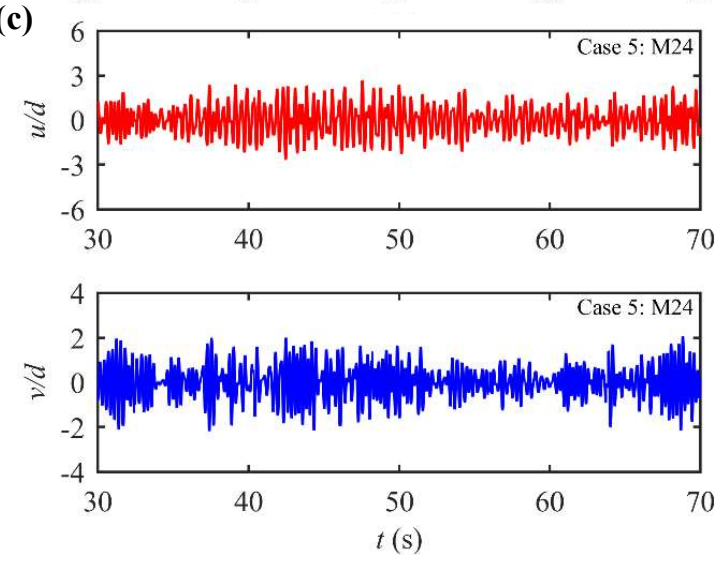

(b)

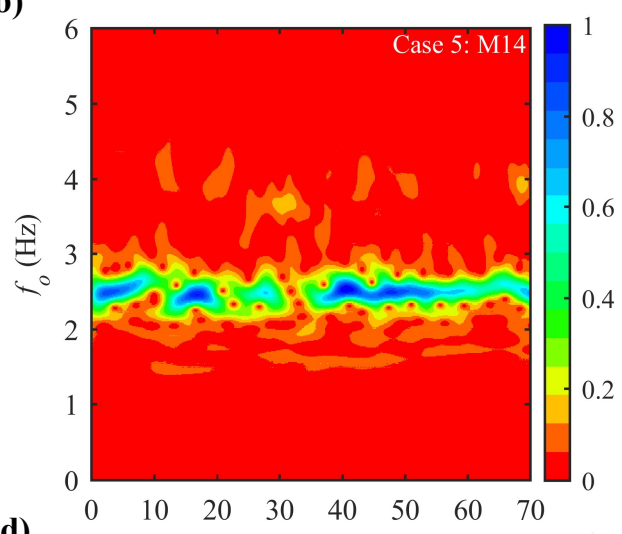

(d)

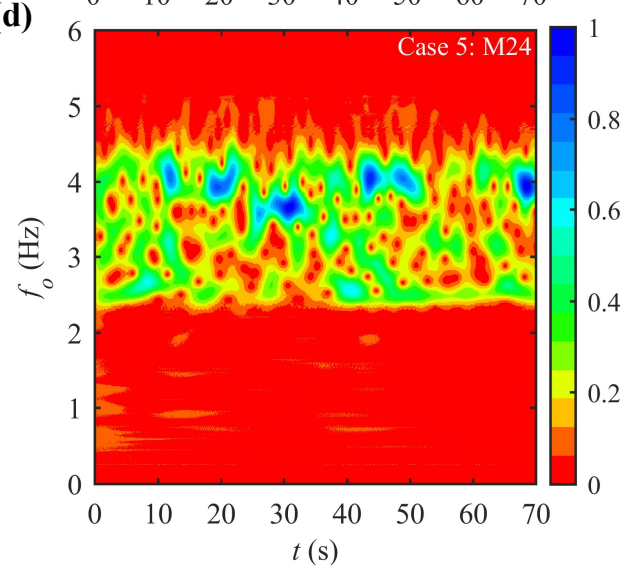

Figure 4. Time histories of riser responses with associated wavelet contour plots at (a, b) M14 and (c, d) M24 for case C5. 
(a)

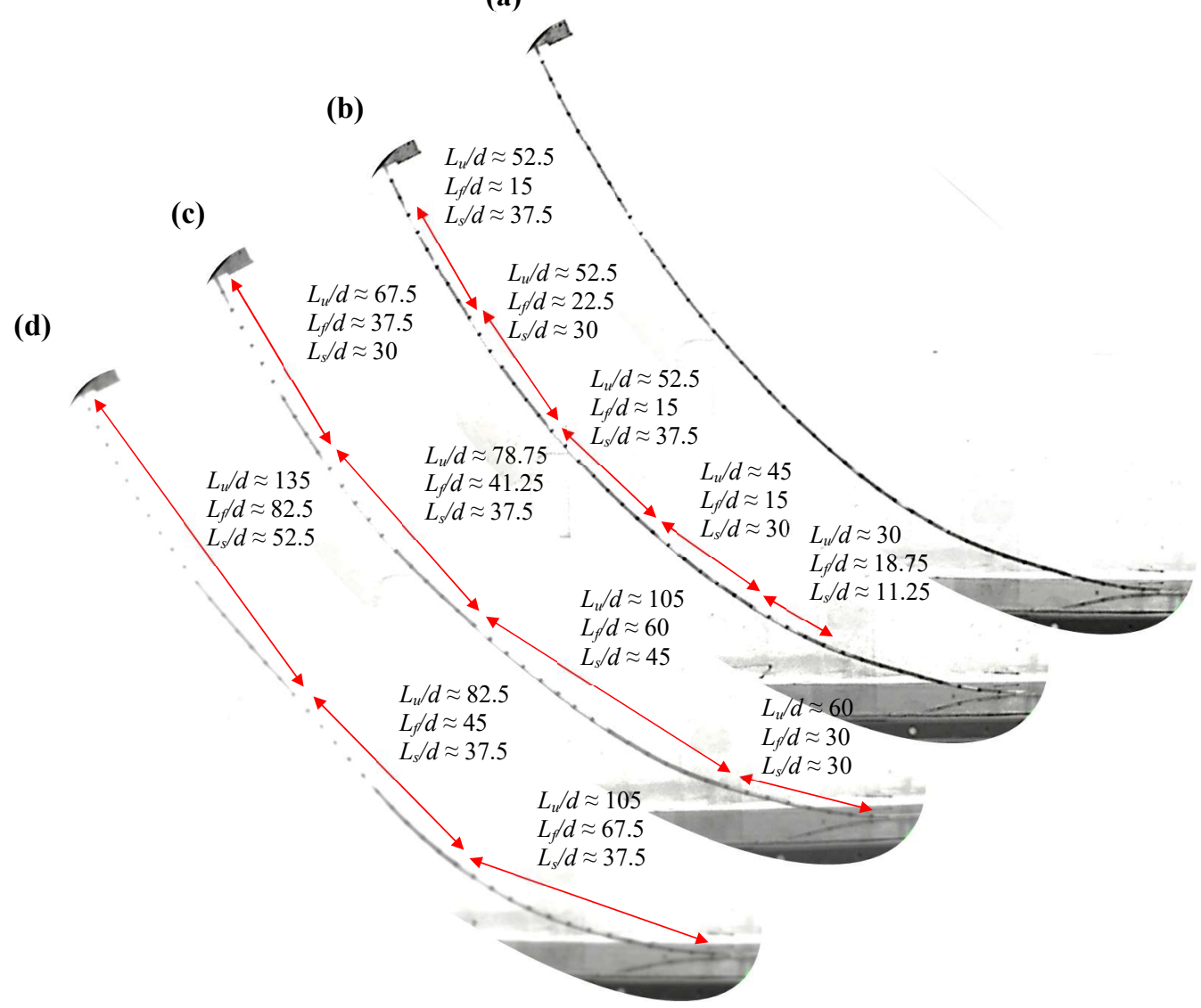

Figure 5. Snapshots of flow regimes for cases (a) C2, (b) C3, (c) C4 and (d) C5: red arrows illustrate the slug units with estimated $L_{u}, L_{f}$ and $L_{s}$.

(a)

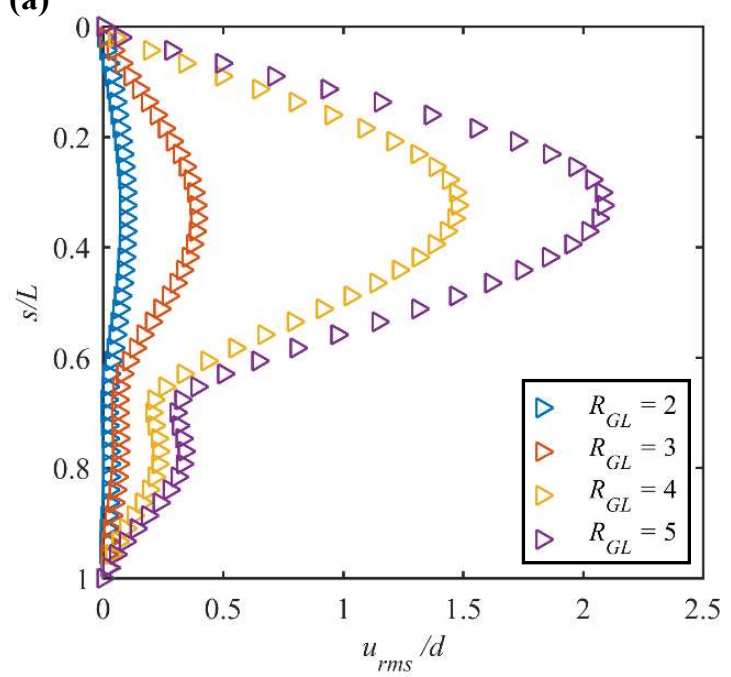

(b)

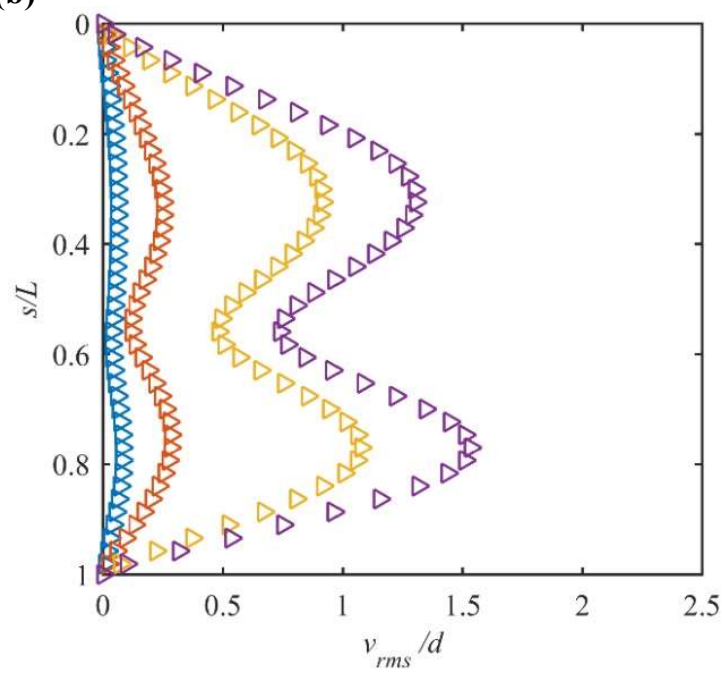

Figure 6. Spatial distribution of RMS responses for cases C2-C5 in (a) $X$ and (b) $Y$ directions.

On the other hand, more scattered frequency contents can be seen at M24. It is interesting to notice that the governing $f_{o}$ becomes about $4.1 \mathrm{~Hz}$ accompanied by co-existing frequencies from $2.5-5 \mathrm{~Hz}$. This dominant $f_{o}$ suggests the occurrence of higher-order modal SIV and justifies the observation of $2^{\text {nd }}$ modal oscillation with small amplitudes in Fig. 3. These features reveal the multi-modal SIV behaviour being closely related to the transient slug flow characteristics.

To further visualize SIV, the flow regimes are illustrated by showing some snapshots of the recorded videos. Fig. 5 displays four frames representing the detailed slug distribution for C2-C5. These instantaneous frames are selected within 40 $<\mathrm{t}<43 \mathrm{~s}$, where the riser responses are pronounced. Note that $\mathrm{C} 1$ result is not shown due to its nearly negligible SIV. Also, the associated RMS profiles in $X$ and $Y\left(u_{r m s}\right.$ and $\left.v_{r m s}\right)$ directions are presented in Fig. 6. It is seen from Fig. 5a that a relatively uniform flow with small bubbles entrained in the liquid phase occurs in $\mathrm{C} 2$. Such a flow regime may result in 
the small dynamic effect as in the single-phase flow case [16]. Thus, it is reasonable to see much smaller riser responses for $\mathrm{C} 2$ in Fig. 6. The two-phase flow patterns reveal slug flows in C3-C5 cases (Figs. 5b-d), with alternative distributions of liquid- and gas-dominant sections.

As for the slug parameters, the slug flow unit length $\left(L_{u}\right)$, liquid slug length $\left(L_{s}\right)$ and elongated bubble length $\left(L_{f}\right)$ are evaluated with respect to $d$ as presented in Fig. 5. It can be seen that the riser tends to accommodate the longer $L_{u}, L_{s}$ and $L_{f}$ as $R_{G L}$ is increased. However, a more regular slug flow takes place at a lower $R_{G L}$. For instance, the difference between the longest and shortest $L_{u} / d$ is about 52.5, 45 and 22.5 for C3-C5 cases, respectively. By inspecting the slug flow regimes (Fig. 5) against the riser SIV (Fig. 6), an increasing trend is revealed from $\mathrm{C} 3$ to $\mathrm{C} 5$ as $L_{u} / d$ increases. A similar experimental observation was also reported in Zhu et al. [12]. Such a correlation may be owing to the enhanced imbalance in the flow state at higher $R_{G L}$, potentially leading to the greater slug flow-induced loads. In contrast, it is reasonable to see that the slug flow with a short $L_{u}$ tends to affect the riser in a timeaveraged manner.

The translational slug unit velocity plays a significant role in determining SIV. In the present study, the higher $R_{G L}$ is achieved by increasing $u_{g s}$ so that the slug flow tends to travel through the riser at a higher unit velocity. As the centrifugal force is related to the flow speed and the curvature, the sagged riser would be subject to greater slug flow-induced loads at higher $R_{G L}$. This key observation reveals a strong correlation among $R_{G L}$, the slug characteristics and the resultant SIV.

Apart from the riser dynamics, local pressure values at the inlet and outlet are also measured. In association with Fig. 6 , the standard deviation of the time-varying pressure $\left(P_{s t d}\right)$ at the two ends, and the riser inlet-to-outlet pressure drop $(d P)$ are displayed in Fig. 7. Higher $P_{s t d}$ are observed as $R_{G L}$ is increased, signalling the strongly increased fluctuation of the pressure from $\mathrm{C} 2$ to $\mathrm{C} 5$ cases. The greater $P_{\text {std }}$ may also be associated with the observed larger $L_{f}$ and $L_{u}$, which consequently result in the amplified SIV [17]. On the other hand, $d P$ tends to increase with $R_{G L}$. It is widely recognized that $d P$ is partially led by the fluid-wall friction forces [18]. For a higher $R_{G L}$, the increasing $d P$ may be due to the larger friction force caused by the higher travelling velocity of the slug flow.

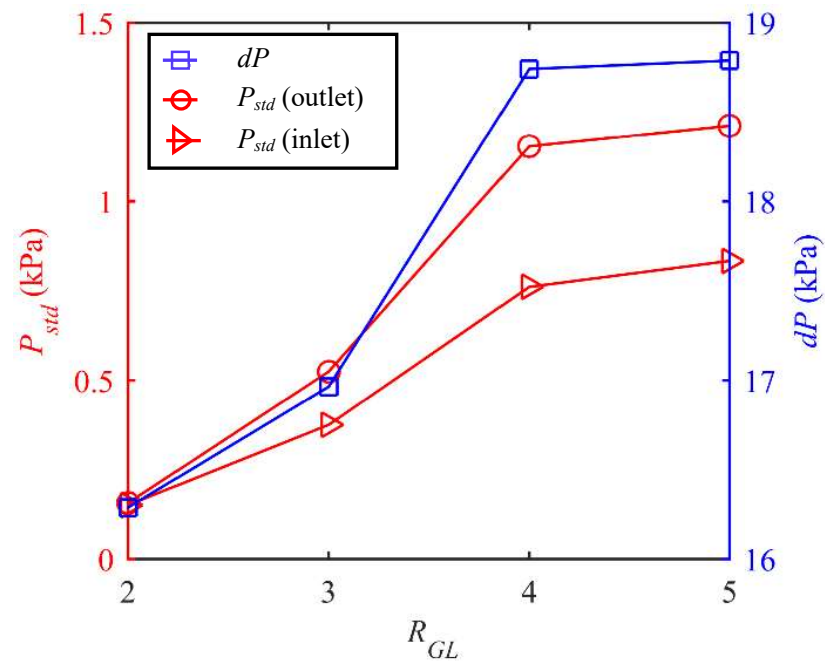

Figure 7. $P_{\text {std }}$ of (red circles) riser outlet and (red triangles) inlet with associated $d P$ at $R_{G L}=2-5$.

\section{CONCLUSIONS}

An inclined sagged riser conveying gas-liquid flows has been experimentally investigated. The experiment tests have been carried out in an air-water test loop with a hanging tube section made of silica gel. Both pipe SIV responses and gasliquid flow patterns have been recorded simultaneously using non-intrusive high-speed cameras. Pressure variations have also been measured at the riser inlet and outlet by pressure transducers. The flow-transporting system has been tested by employing different gas-liquid flow velocities.

Occurrence of slug flows is captured at the relatively high ratios of the gas to liquid superficial velocities rendering a large-amplitude SIV of the flexible riser. Under different flow conditions, the slug flow characteristics are observed to vary significantly in terms of the travelling velocities and lengths of different slug units. These entail unsteady SIV with modulated amplitudes and frequencies. The fundamental planar mode is found to prevail in the SIV of the present riser, whereas coexisting higher mode is also observed at different locations. Significant corrections between the slug flow and SIV are revealed from laboratory tests. By increasing the superficial gas-to-liquid velocity ratio, pronounced SIV responses are noticed. This is related to the fast travelling slug flows with long slug unit lengths, enhancing the slug flow-induced momentum forces. These observations highlight the effects of superficial gas-liquid superficial velocities on the slug flow characteristics and the resultant SIV behaviours.

\section{ACKNOWLEDGEMENTS}

The authors thank the funding support from the Engineering and Physical Sciences Research Council (EPSRC) of the UK Research and Innovation through the "MUltiphase Flowinduced Fluid-flexible structure InteractioN in Subsea applications (MUFFINS)" project grant EP/P033148/1 for the numerical model, post-processing and analysis parts, and the National Natural Science Foundation of China through the grant no. 51979238 for experimental research facilities.

\section{NOMENCLATURE}

$\begin{array}{ll}d(D) & \text { Riser internal (outer) diameter } \\ d P & \text { Inlet-outlet pressure drop } \\ f_{n}\left(f_{o}\right) & \text { Riser natural (oscillation) frequency } \\ L & \text { Riser length } \\ L_{f} & \text { Elongated bubble length } \\ L_{s} & \text { Liquid slug length } \\ L_{u} & \text { Slug unit length } \\ P_{s t d} & \text { Standard deviation of internal pressure } \\ R_{G L} & \text { Superficial gas-liquid velocity ratio } \\ \mathrm{RMS} & \text { Root-mean-squared } \\ s & \text { Arclength coordinate } \\ T & \text { Acquisition time } \\ t & \text { Time } \\ u_{g s} & \text { Superficial gas velocity } \\ u_{l s} & \text { Superficial liquid velocity } \\ u\left(u_{r m s}\right) & \text { Horizontal (root-mean-squared) riser } \\ v\left(v_{r m s}\right) & \text { displacement } \\ X(Y) & \text { Vertical (root-mean-squared) riser } \\ \Delta u & \text { displacement } \\ \Delta v & \text { Horizontal (vertical) direction } \\ & \text { Riser horizontal displacement change } \\ & \text { between images } \\ & \text { Riser vertical displacement change } \\ & \text { between images }\end{array}$




\section{REFERENCES}

[1] DNVGL-ST-F201, 2018, "STANDARD," Dynamic risers. [2] Hara, F., 1977, "Two-phase-flow-induced vibrations in a horizontal piping system," Bulletin of JSME, 20(142), pp. 419427.

[3] Patel, M. H., and Seyed, F. B., 1989, "Internal flowinduced behaviour of flexible risers," Engineering Structures, 11(4), pp. 266-280.

[4] Ma, B., and Srinil, N., 2018, "Dynamic characteristics of deep-water risers carrying multiphase flows," ASME 2018 37th International Conference on Ocean, Offshore and Arctic Engineering, pp. OMAE2018-77381.

[5] Chatjigeorgiou, I. K., 2017, "Hydroelastic response of marine risers subjected to internal slug-flow," Applied Ocean Research, 62, pp. 1-17.

[6] Ortega, A., Rivera, A., and Larsen, C. M., 2018, "Slug flow and waves induced motions in flexible riser," Journal of Offshore Mechanics and Arctic Engineering, 140(1).

[7] Safrendyo, S., and Srinil, N., "Slug flow-induced oscillation in subsea catenary riser experiencing VIV," Proc. ASME 2018 37th International Conference on Ocean, Offshore and Arctic Engineering, pp. OMAE2018-77298.

[8] Al-Hashimy, Z. I., Al-Kayiem, H. H., and Time, R. W., 2016, "Experimental investigation on the vibration induced by slug flow in horizontal pipe," ARPN J. Eng. Appl. Sci, 11, pp. 12134-12139.

[9] Wang, L., Yang, Y., Li, Y., and Wang, Y., 2018, "Dynamic behaviours of horizontal gas-liquid pipes subjected to hydrodynamic slug flow: Modelling and experiments," International Journal of Pressure Vessels and Piping, 161, pp. $50-57$.
[10] Liu, G., and Wang, Y., 2018, "Study on the natural frequencies of pipes conveying gas-liquid two-phase slug flow," International Journal of Mechanical Sciences, 141, pp. 168-188.

[11] Mohmmed, A. O., Al-Kayiem, H. H., Nasif, M. S., and Time, R. W., 2019, "Effect of slug flow frequency on the mechanical stress behavior of pipelines," International Journal of Pressure Vessels and Piping, 172, pp. 1-9.

[12] Zhu, H., Gao, Y., and Zhao, H., 2019, "Experimental investigation of slug flow-induced vibration of a flexible riser," Ocean Engineering, 189, p. 106370.

[13] Vieiro, J. J., Akhiiartdinov, A., Sævik, S., Larsen, C. M., and Nydal, O. J., 2019, "Two-way coupled fluid-structure interaction of gas-liquid slug flow in a flexible riser: smallscale experiments and simulations," Multiphase Science and Technology, 31(1).

[14] Spedding, P. L., and Nguyen, V. T., 1980, "Regime maps for air water two phase flow," Chemical Engineering Science, 35(4), pp. 779-793.

[15] Issa, R. I., and Kempf, M. H. W., 2003, "Simulation of slug flow in horizontal and nearly horizontal pipes with the two-fluid model," International Journal of Multiphase Flow, 29(1), pp. 69-95.

[16] Paidoussis, M. P., 2014, Fluid-Structure Interactions. Volue 1, Slender Structures and Axial Flow, Amsterdam : Acaemic Press.

[17] Ma, B., and Srinil, N., 2020, "Planar dynamics of inclined curvd flexible riser carrying slug liquid-gas flows," Journal of Fluids and Structures, 94, 102911.

[18] Taitel, Y., and Barnea, D., 1990, "Two-Phase Slug Flow," Advances in Heat Transfer, 20, pp. 83-132. 